\title{
Algunas consideraciones lingüísticas para el estudio del desarrollo histórico del sujeto y del yo
}

\section{Carlos Gustavo González*}

\section{Resumen}

Como inicio a una contribución al desarrollo psicológico-histórico del sujeto, es analizado el yo como agente. Utilizando métodos de lingüística comparativa y de antropología lingüística fueron identificados elementos sintácticos que pueden utilizarse como indicadores del desarrollo del yo. Fundamentalmente, se analizan las alteraciones en las voces verbales pasivas, media y activa, porque implican cambios en el modo de actuar del yo. Volviendo breve-

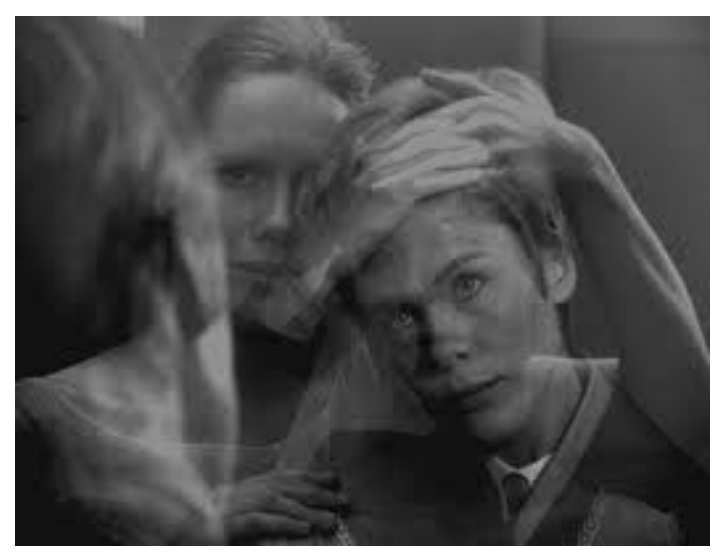
mente al análisis de Dodds, se presentan después ejemplos similares de cambios en el uso de las voces verbales en latín y en inglés. Intentando re-interpretar estos cambios de la agencia del yo en el aparato psíquico, se propone el concepto de "coagentes del yo".

Palabras clave: agente; yo; sujeto; linguística; filosofía

\section{Abstract}

As a initial contribution to the historical-psychological development of the subject, the ego is analyzed in its role of agent. Using methods of comparative linguistics and linguistic anthropology, are identified syntactic constructs that can be used as indicators of ego development. Fundamentally, the changes in the passive, middle and active verbal voices are analyzed, since they imply changes in the ego's way of acting. Turning briefly to Dodds's analysis, similar examples of changes in the use of verbal voices in Latin and English are presented below. Trying to reinterpret these ego agency changes in the psychic apparatus, the concept of "co-agents of the ego" is proposed.

Keywords: agent; I; subject; linguistics; philosophy

* Doctor en Lógica y Filosofía de la Ciencia por la Universidade Estadual de Campinas (Unicamp), Brasil. Profesor investigador en la Universidade Federal de Uberlândia, Brasil. Tiene publicaciones en las áreas de Filosofia, Lógica y Computación. Curriculum Vitae: http://lattes.cnpq.br/2262227235584491 Mail : gonzalcg@gmail.com 
Las modificaciones lingüísticas son modificaciones del estado del sujeto -de su relación con el cuerpo, con los demás, con los objetos. (Kristeva, 1981, p. 13)

\section{Introducción}

La preocupación por el desarrollo histórico del sujeto ha sido expresada por diversos autores. $\mathrm{Si}$, por una parte, un sujeto concebido de una manera idealista y atemporal puede permanecer estático y sin modificaciones, en cambio, concebido inmerso en el flujo de la historia es natural pensar que los acontecimientos históricos tengan una influencia en él y que el sujeto mismo tienda a modificarse para dar cuenta de las constantes nuevas condiciones que crea cada vez la historia.

En los días de hoy, debido a los desarrollos de diferentes autores del psicoanálisis, cuando se presta atención al sujeto, nos encontramos con la estructura y funciones del aparato psíquico humano. De esta manera debemos dar cuenta, para nuestro estudio, como contexto, esas ideas del aparato psíquico. En consecuencia, este artículo se concentró en el yo y sus funciones; en particular, en la función de agente del yo pasó en primer lugar, por haber sido encontradas en manifestaciones lingüísticas que parecen ser indicativas del cambio histórico en la agencia del yo.

Por lo tanto, el objetivo principal de este estudio es contribuir al entendimiento del desarrollo de la agencia del yo como una parte fundamental del desarrollo histórico del sujeto.

La sección 2 pretende mostrar una visión simplificada y general,resaltando algunas interpretaciones del concepto del yo. La Sección 3 explicará el yo agente y mostrará evidencia histórica del desarrollo del yo a través de estudios lingüísticos y de la antropología lingüística. La sección 4 presentarálas conclusiones preliminares de este estudio en marcha, fundamentalmente como el concepto de "intervención externa" de Dodds puede ser internalizado como coagentes del yo.

\section{El concepto de yo}

Diferentes conceptos del yo y diferentes enfoques disciplinarios llevaron a una pluralidad de significados que es difícil determinar totalmente. Sin embargo, algunas de esas modalidades pueden listarse:

El yo como:

1. agente. El yo-agencia;

2. representación en la autoconciencia, lo que Kant denomina apercepción;

3. la totalidad do individuo, o si-mismo, referido como self en inglés;

4. una entidad metafísica, a veces identificado con el alma;

5. el aparato yoico, un subaparato del aparato psíquico;

$$
\text { ! [posiblemente más significados] }
$$

Veamos un primer esbozo de esos sentidos:

El yo agente: En un primer caso, podemos considerar la función del yo en la voz activa del verbo:

-Yo camino.

—Yo quebré el vaso.

El agente es muchas veces entendido como la causa: mi acción fue la causa de que el vaso esté quebrado. A partir de esa causalidad pueden ser establecidas otras cosas, como consecuencias de la acción, responsabilidad, etc. Vamos a denominar agencia a este carácter de agente del yo.

La apercepción del yo: Cuando realizados una acción, cuando conocemos, cuando 
somos pasivos de una acción externay en otros casos, tenemos una imagen o representación de nosotros mismos acompañando la acción. Por ejemplo, si quebramos un vaso, nos vemos a nosotros mismos quebrando el vaso.

El si-mismo: Este concepto es usado con gran ambigüedad en la literatura, como sucede con "self" en la lengua inglesa. El uso más general, -entiendo-es cuando se refiere a la totalidad física y psíquica de la persona. Por ejemplo, si una persona se hirió una pierna, podría decir "tengo una herida en la pierna”, pero también podría usar el yo en la función de si-mismo y exclamar; "yo tengo una herida en la pierna”. Si una persona no vio un pequeño perro en el suelo y lo pateo sin querer, podría decir: "fui yo (mismo) quien pateo el perro, pero sin darme cuenta".

Elyo metafísico: Si un pensador asume una posición realista que implique algún concepto metafísico de realidad, entonces el yo que interacciona con esa realidad tiene, en algún sentido, un carácter metafísico. Si el yo no pasase de una entidad psíquica o de un objeto físico no tendría condición de llegar a "la realidad en sí misma”. En el caso de posiciones místicas, religiosas, espiritualistas, etc., puede pasar que el yo tenga un carácter más allá de lo físico, por ejemplo, como alma, espíritu, etc.

El pasaje de la res cogitans a la res extensa en Descartes-objeto de muchas discusionespuede ser interpretada en el sentido de un pasaje de una entidad puramente metafísica, la res cogitans, para otra entidad que tiene, por lo menos parcialmente, un carácter físico, la res extensa.

\section{El yo agente y paciente}

La manera más simple de concebir un agente es como causa u origen de una acción. Tradicionalmente, esta acción puede no tener un objeto -los verbos intransitivos-como por ejemplo "correr". Si, por el contrario, la acción tiene un objeto-los verbos transitivos-como "pintar", vamos a denominar "paciente" al objeto que recibe la acción. A lo largo de este artículo veremos cómo este esquema simple puede complicarse. Por ejemplo:

La identificación del sujeto como agente o paciente puede también estar vinculada al contexto pragmático, como es el caso de este ejemplo, citado por [Pontes, 1986]: "João arrancou um dente hoje" ["Juan se sacó un diente hoy"]. Siendo Juan el dentista, él será probablemente el sujeto agente, pero, si no es el dentista, Juan es apenas otro de los pacientes que fue sometido a un tratamiento dental; entonces, Juan ya no es el sujeto agente y, sí, el sujeto paciente. (de Carvalho \& Pria, 2010, p. 79)

Varios autores del área del psicoanálisis ${ }^{1}$ analizaron la evolución del yo considerando la visión y la imagen, que son la base del estudio del narcisismo y del mito de Narciso. Pero el lenguaje y la comunicación lingüística también tienen importancia en la evolución del yo.

Recordemos que el carácter o la propiedad de ser agente es referida con la palabra "agencia" ${ }^{2}$. Según un importante autor de la antropología lingüística, A. Duranti:

Un tipo básico y recurrente de agencia, expresadoy percibido por ellenguaje, es lo que podríamos llamar "auto-" o "yo-afirmante". Este tipo de agenciageneralmente se alcanza, aunque en grados diferentes, siempre que el lenguaje es utilizado. El propio acto de

1 Notablemente Lacan con la "etapa del espejo" [Lacan, 1966] y Andre Green estableciendo la importancia del narcisismo en la formación del yo.

2 [...] se denomina agencia a la capacidad que posee un agente (una persona u otra identidad) para actuar en un mundo". Ver https://es.wikipedia. org/wiki/Agencia_(filosof\%C3\%ADa), accedido el 13/06/2019. 
hablar ante otras personas que pueden percibir tal acto, establece al hablante como un ser cuya existencia debe ser tenida en cuenta en términos de sus objetivos y habilidades comunicativas. ${ }^{3}$

En otras palabras, según Duranti, cada vezque ellenguaje se usa en público, frente alos demás, aparece un tipo de yo agente, el yo-afirmante o auto-afirmante. Tal vez las formas primitivas del yo tengan quever con eso, sobre todo la aparición de la palabra para referirse a la primera persona del singular: "ego", “yo”. Lejos de surgir de un soliloquio, ese proceso implica reconocer al otro como un agente y luego auto-afirmante como agente delante de los demás.

Es de notar que el mito de Narciso describe a un joven que se aísla huyendo del contacto romántico. Él muere mirando su propia imagen en el agua, sin ninguna otra persona, en un estado de aislamiento. Lo que vemos en las palabras de Duranti es un hombre hablando con otros, en un contexto social y no de aislamiento.

Otro autor que se opone a concebir el surgimiento y maduración de yo en un contexto de aislamiento, es el teórico del psicodrama J. L. Moreno, según el cual:

El rol aparece aún antes de que surja el yo. No son los roles quienes emergen del yo, sino el yo quien puede emerger de los roles. (Cukier, 2005, p. 252)

El punto de contacto con la concepción del concepto de yo en Moreno es inmediata: el yo surge del habla inducida por los roles que el individuo tiene en la sociedad.

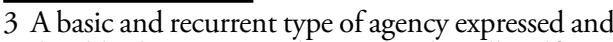
realized by language is what we might call "self-" or "ego-affirming." This type of agency is usually achieved, albeit in different degrees, any time language is used. The very act of speaking in front of others who can perceive such an act establishes the speaker as a being whose existence must be reckoned with in ter$\mathrm{ms}$ of his or her communicative goals and abilities. ([Duranti, 2004a, p. 455)
}

Si sucede, como escribe Julia Kristeva, que los estudios lingüísticos del siglo XX han concebido el lenguaje como una entidad separada y estática - y por lo tanto, deshumanizaron el lenguaje- no puede sorprender que las investigaciones de la antropología lingüística, como las de Duranti, indiquen el camino de un lenguaje humanizado, que Kristeva concibe como parte de un proceso dinámico de significación.

\subsection{Declinaciones, pronombres personales y agencia}

Con el fin de que este artículo sea auto-contenido, se ofrecerán a continuación algunas nociones básicas sobre ciertos aspectos de diferentes lenguajes.

Las lenguas antiguas, latín y griego, usan declinaciones para sustantivos, adjetivos y pronombres. De manera análoga a la conjugación de los verbos, los sustantivos y adjetivos (cuando eran regulares) mantenían una raíz, pero cambiaban la terminación según la función gramatical, lo que se denomina "caso". $\mathrm{El}$ adjetivo, además de tener que coincidir con el sustantivo que modifica en género y número, también tiene que coincidir con el caso. Por ejemplo, al sustantivo núcleo del sujeto gramatical correspondía el caso nominativo ${ }^{5}$ :

\section{—Mensa est alba (La mesa es blanca)}

Pero si el sustantivo " mensa " está formando parte del objeto gramatical, el caso que debe ser usado es el acusativo y la terminación debe sumar la letra "m":

4 Por ejemplo, McAfee escribe: "En lugar de tratar la lenguaje como una entidad separada y estática, Kristeva ha visto las partes de un proceso significante dinámico.", ("Instead of treating language as a separate, static entity, Kristeva has seen it as part of a dynamic signifying process.") [McAfee, 2004], p. 14.

5 Otras veces por el dativo agente, como en la voz pasiva. 
blaideo albam mensam (Yo veo la mesa

Nótese que la función de agente era representada frecuentemente por el nominativo. ${ }^{6}$ Para de paciente, el que recebe la acción indicada por el verbo, era usado el acusativo.

El latín tenía un total de seis casos. En el camino que va desde el latín hasta las lenguas modernas, los casos de sustantivos y adjetivos se perdieron la mayoría de las veces. Mientras la lengua culta continuaba siendo el latín, lenguas regionales fueron apartándose del latín y tomando sus propias características, unas diferentes de otras.

Cada una de las lenguas romances al separarse del latín, tomó como base uno o dos casos: algunas tomaron como base el nominativo, otras el acusativo, etc., perdiendo la declinación. Para el plural, el castellano tomó como base fundamentalmente el acusativo. Como el acusativo plural del latín generalmente agrega la letra "a", eso fue heredado por el español en sus plurales: poema, poemas -mesa, mesas. En el italiano ese camino fue más complexo, tomando muchas veces el plural del nominativo, que en latín tiene más formas que los plurales del acusativo. como poema, poemi. Mientras esto sucedía con sustantivos y adjetivos, los pronombres personales conservan, hasta el día de hoy, formas parciales de declinaciones:

— Yo empujé la mesa.

—Juan me empujó.

Siendo "yo" el nominativo y "me" el acusativo.

Como los casos señalan el papel de agente o paciente, de alguna manera, abandonar la referencia explícita a esos papeles fue más difícil para los pronombres personales, que representan personas, que para cosas inanima-


pasiva. das. De esta manera, conservamos hasta hoy formas parciales de declinación de pronombres, que en el caso del nominativo y el acusativo, "yo" y "me", representa la mayoría de las veces la diferencia entre las funciones de agente y paciente.

Este tratamiento especial delos pronombres no es un caso aislado. Duranti afirma:

[...] en Guaraní y Lakota el primer pronombre personal singular tiene dos formas. Uno se utiliza para asuntos de verbos intransitivos de acciones (por ejemplo, yo voy, yo levanto) y verbos transitivos (por ejemplo, yo lo traigo, yo lo tomo) y la otra forma se utiliza para cuestiones con verbos de estado (por ejemplo, yo estoy enfermo, yo estoy con sueño) y para el objeto directo (objeto / paciente) de verbos transitivos (por ejemplo, él me llevará, él me va a matar ${ }^{7}$

(Duranti, 2004a, p. 462)

Grosso modo, una forma del pronombre personal corresponde al agente y otra al paciente.

\subsection{Voces activa, media y pasiva de verbos y verbos deponentes}

Voy a hacer algunas descripciones gramaticales, muchas veces consideradas básicas y enseñadas en los primeros años de la escuela para servir de contexto y por la sistematicidad de las ideas y unificación del vocabulario técnico. Los desarrollos en este sentido pueden encontrarse en la literatura en diferentes presentaciones de gramáticas en diversas lenguas.

\footnotetext{
$7[\ldots]$ in Guarani and Lakhota the first singular personal pronoun has two forms. One is used for subjects of intransitive verbs of actions (e.g. I go, I get up) and transitive verbs (e.g. I bring it, I catch it) and the other form is used for subjects of stative verbs (e.g. I am sick, I am sleepy) and for the direct object (Object/Patient) of transitive verbs (e.g. it will carry me off, he'll kill me).
} 
En lenguas de la familia de las indoeuropeas, pero no exclusivamente, la flexión de los verbos reconoce, para algunos de ellos, voces. Por ejemplo, en español se hace una distinción entre las voces activa, pasiva y reflexiva. Las diferentes lenguas no siempre utilizan los mismos estándares para el análisis gramatical, de modo que los estudios comparativos, como éste, a menudo se ven obligados a abusos de lenguajes o alejamiento de las denominaciones y enfoque típico en la gramática de una lengua. Por ejemplo, el inglés reconoce el presente continuo como tiempo verbal, pero el análogo no es reconocido como tiempo verbal en portugués y español. En latín y griego se habla de voces activa, media y pasiva. Voy a usar "voz media”, para unificar la nomenclatura, también para referirme a la voz reflexiva, diferente de los términos utilizados en las gramáticas de portugués y español.

En el uso típico, en la voz activa el sujeto es el agente de la acción del verbo y el predicado tiene alguna construcción, como el objeto, donde está el paciente que recibe esa acción. Por ejemplo:

\section{- Juan quebró el vaso.}

Pero en la voz pasiva, el paciente aparece en el sujeto y el agente en alguna construcción del predicado:

\section{—El vaso fue quebrado por Juan.}

La voz media es usada muchas veces cuando el agente es también el paciente, recibiendo la acción del verbo

\section{— Juan se peinó.}

Las voces media y pasiva se pueden utilizar en otros sentidos: "se usan mayúsculas en la primera letra de una frase" o "se vende". Como otros usos de las lenguas, las voces pueden estar ligadas a matices psicológi- cos. Por ejemplo, si una persona rompió un vaso mientras lo estaba lavando, para negar responsabilidades podría decir:

—El vaso se rompió.

Negándose él mismo como agentey colocando la voz media casi como si el agente fuera el mismo vaso, cosa que puede ser coherente con la manera de pensar de un nińo de 6 ańos. Los matices de otros usos voz media son curiosos, como en el español "se arrepintió".

Es común en griego y en latín catalogar algunos verbos como deponentes. Estos verbos a pesar de ser conjugados o en la voz media o en la voz pasiva en la lengua original, son traducidos usando la voz activa. Como es típico colocar el significado conocido o de la propia lengua como absoluto-una manera de desconocer la diversidad cultural-es común encontrar expresiones como la siguiente:

Los verbos deponentes tienen un significado activo, pero sólo formas media (o media y pasiva) ${ }^{8}$

Lo correcto sería decir: "tienen un significado activo para nosotros", pues si el verbo era usado en la voz pasiva-por ejemplo, por los griegos- entonces es de suponer que para ellos tenía a menudo un significado pasivo, o lo tuvo en algún tiempo. De esta manera, es posible que una acción para la cual en una cultura el yo tiene una actitud paciente, tenga, en otra cultura, el yo en una función activa de agente.

Por lo tanto, sería más apropiado decir que algunas situaciones en las que una lengua, por ejemplo el griego clásico, usa la voz media o pasiva, nuestra lengua usa la voz activa. Dada la importancia que la agencia tiene para la vida social y para la ética, sería muy

8 "Deponent verbs have an active meaning but only middle (or middle and passive) forms." [Smyth, 1956], p. 107. 
curioso que los griegos usasen los verbos confundiendo frecuentemente ${ }^{9}$ quién es agente y quién no lo es. ${ }^{10}$ Como ejemplo de un verbo deponente, en la Ciropedia de Jenofonte, podemos leer:

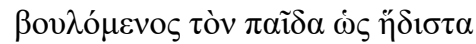 $\delta \varepsilon 1 \pi v \varepsilon \tilde{i} v$}

Queriendo que el niño cene lo más placentero posible. ${ }^{11}$

Sin embargo, el verbo "querer" ( $\beta$ ov $\lambda$ ó $\mu \alpha \tilde{\imath}$ ) está en la voz media, la que debería ser "queriéndose", dando la idea de agente y paciente: en cierta medida la persona padece la voluntad, es también paciente.

En latín también encontramos verbos deponentes:

La siguiente lista de verbos deponentes y semi-deponentes del latín no está, en modo alguno, completa; pero un número suficiente atestigua su naturaleza: deponentes / hortor (animar), liceor (ofrecer precio), mereor (ganar, obtener), misereor (tener pena), blandior (adular, persuadir), mentior (mentir) [siguen más 14 verbos]. ${ }^{12}$

9 Sin embargo, debemos considerar que los cambios lingüísticos no son uniformes. Por ejemplo, algunas expresiones sobreviven, de forma análoga a los "fósiles vivientes" en evolución. Pero hablar de una manera general de verbos deponentes con una concepción como la de la nota anterior implica suponer que de forma sistemática los hablantes de lengua griega antigua se equivocaban al concebir el agente.

10 Como un ejemplo de rechazo de la atribución de una falsa agencia, tenemos el proverbio espańol "aramos dijo el mosquito". Ver, por ejemplo, (Lombardini, 2010, p. 132)

11 Xenofonte, Ciropédia, 1.3.4. Texto grego em: http://www.perseus.tufts.edu/hopper/text?do$\mathrm{c}=$ Xen.+Cyrop.+1.3.4\&fromdoc=Perseus\%3Atext \%3A1999.01.0203.

12 The following list of Latin deponent and semi-deponent verbs is by no means complete; however, a sufficient number attest to their nature: deponent / hortor (to encourage), liceor (to
Para analizar los aspectos psicológicos envueltos, precisamos volver a considerar la pasaje de La Ilíada donde figura la defensa de Agamenón.

\subsection{Volviendo a la Defensa de Agamenón}

En González, (2019) se analiza el pasaje dela La Iliada donde Agamenón se defiende porque, cegado por Ate, quitaaAquiles su esclava Brunilda. Esteestudio toma como referencia (Dodds, 1951). Agamenón se defiende diciendo que obró así porque su entendimiento había sido ofuscado por Ate ('A $\tau \eta)$.

[...] pero yo no soy la causa, sino Zeus, la Moira y las Eríneas que vagan en la oscuridad, los cuales en público me pusieron en el corazón la salvaje Ate, por la cual un día saqué de Aquiles su premio. (Ilíada, 19.85-88)

Dodds discute largamente el significado de la palabra griega Áte ('A $\tau)$ ). Esta palabra tiene dos significados fuertemente asociados: como una diosa y como un estado psíquico. Dentro de ese universo semántico tan rico, aparecen diferentes maneras de indicar la sumisión del yo y la pérdida de su agencia: irresponsabilidad, impulsividad, falta de deliberación, etc. El carácter de una fuerza externa que se impone al yo aparece señalada de diferentes maneras. Dodds ofrece una definición de "ate":

[...] ate es un estado de la mente -una ofuscación o confusión temporaria de la conciencia normal. ${ }^{13}$

Resalta el carácter externo al yo que tiene ese proceso, proponiendo la expresión intervención psíquica (psychic intervention). La interpretación psicológica de (González, 2019) ve este análisis de Dodds desde el punto

offer a price for ), mereor (to earn, obtain), misereor (to pity), blandior (to flatter, coax), mentior (to lie). [Sabatini, 1979, p. 152)

13 ate is a state of mind -a temporary clouding or bewildering of the normal consciousness. (Dodds, 1951, p. 5.) 
de vista de una internalización ${ }^{14}$, partiendo del presupuesto de que el aparato psíquico tiene un sub-aparato yoico. Además del yo, también forman parte del aparato yoico: el superyó, el yo-ideal y el ideal-yo. Estos últimos son coagentes del yo, en el doble sentido de co-agente, "actuar con" el yo, y el de forzar, coaccionar el yo. Pero estas ideas están en una forma hipotética y envolverán investigaciones futuras. De una u otra manera, el papel de agente es quitado parcialmente del yo, sea por pensar que el agente fue una deidad, sea por tener una unidad funcional dentro del aparato psíquico que co-actúa con el yo y lo coacciona, como sucede con el superyó en la teoría freudiana. ${ }^{15}$

\subsubsection{Dela a voz pasiva a la media}

Volvamos a La Iliada donde analizaremos un pasaje para aclarar el funcionamiento de las voces y sus interpretaciones psicológicas: las dos líneas de 19.136-137. Entiendo que en esta cita, Ate, el agente, está personalizado como la diosa griega. El pronombre relativo está en femenino, representando Ate como agente de la voz pasiva, ligado a Ate, quedando:

[Ate] por la cual primero fui enceguecido, mas después me enceguecí y Zeus apartó mi entendimiento. ${ }^{16}$

14 Cfr. con el concepto de internalización en Freud según [de Mijolla, 2005], p. 537, artículo EXTERNALIZATION-INTERNALIZATION.

15 Según (de Mijolla, 2005), (El superyo) resulta esencialmente de la internalización de la autoridad paterna". "It [the superego] results essentially from the internalization of parental authority", Ibid., artículo SUPEREGO, p. 1690ss. De manera similar, la ofuscación de la conciencia, personificada en la diosa Ate, se internaliza en el aparato yoico como una impulsibidad coagente del yo, que puede coaccionar el yo. Para la noción de superyo ver, por ejemplo, (Roth, 2001).

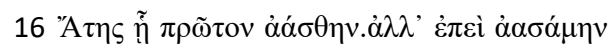

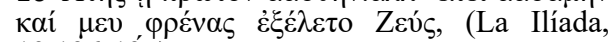
19.136-13́7)
Observemos las tres frases en conjunto. La primera está en voz pasiva, el sujeto es primera persona (Agamenón) y el agente es un pronombre relativo que está por Ate, pues Ate es el agente. En la segunda, el verbo está en voz media, el sujeto es nuevamente la primera persona, Agamenón. En la tercera, el sujeto, Zeus, está en tercera persona, activo. Para entender, mejor voy a hacer una glossa:

"Primero, Ate me impidió razonar. Después me quedé confuso y Zeus sacó mi entendimiento".

Recordemos que en español es también usada la voz reflexiva (= media) en el verbo confundir: "Me confundo". Esta voz media da idea de que el sujeto es tanto agente como paciente. Algo me confundió, yo me confundí.

En la descripción de Sabatini:

[...] un criterio de la voz media del griego: el sujeto es el paciente de la acción del verbo y sufre una reacción interna. ${ }^{17}$

Como conclusión parcial, vemos que, en primer lugar, el sujeto se siente víctima de una intervención externa. El agente es un dios, que actúa en una intervención externa. Pero en el proceso de internalización, el verbo pasa del pasivo al medio, como empezando a adquirir un sentido de activo, lo que significaría que el sujeto ahora se asume parcialmente como agente, en lugar de la intervención externa del dios al mismo momento que sufre un proceso interno. Considero un caso significativo el verbo de voluntad, ( $\beta$ ovió $\mu \alpha \tilde{i}])$, visto en el ejemplo de Jenofonte. Si es así, en

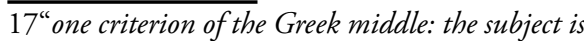
the recipient of the action of the verb and suffers an internal reaction." (Sabatini, 1979, p. 152) las itálicas son mías. 
algún pasado el sujeto sintió que la voluntad, o, por lo menos, algunas voluntades, eran colocadas desde fuera, como intervenciones externas, que poco a poco se fueron transformando en voluntad del sujeto: más específicamente, en voluntades del yo, aumentando su papel de agente. Sin embargo, el sujeto no se siente totalmente agente: cree que en parte es paciente de la acción y por eso es usada la voz media.

\subsection{Cambios de agente en inglés}

Para mostrar que lo que acabamos de analizar en griego no es un caso aislado, vamos a ver cómo en el inglés antiguo también ocurre un cambio en la agencia que es visible en los verbos.

La cuestión ahora surge en cuanto a la naturaleza específica del verbo impersonal en el inglés antiguo y en el inglés medio. Una lectura atenta de la lista de verbos en esa categoría mostrará que esos verbos poseen la naturaleza específica de verbos similares encontrados en los sistemas de la voz media del griego y de deponentes en latín. [...] me repents, me wonders, me likes, me afflicts, me hungreth, me vexes, me thinks (aparece para mí). ${ }^{18}$

Es interesante notar que en el español actual se dice "me arrepiento", "me maravillo" y "me gusta”, pero en portugués se dice "me gusta". Así, el proceso de cambio del agente lejos de ser algo uniforme, pre-

18 "The question now arises as to the specific nature of the impersonal verb in Old and early Middle English. A perusal of the list of verbs in this category will show that these verbs possess the specific nature of similar erbs as found in the Greek middle and Latin deponent systems. [...] me repents, me wonders, me likes, me afflicts, me hungreth, me vexes, me thinks (it appears to me)." [Sabatini, 1979], p. 153.

Transliterando, pero no traduciendo, las formas arcaicas del inglés quedarían: "me arrepientes", "me maravillas", "me gusta", "me aflige", "me hambrea", "me frustra", "me piensa". Notar que expresiones similares de algunas de esas formas son usadas en español. senta notables variantes. Es de suponer que las variaciones psicológicas y culturales en distintos pueblos tengan mucho a ver con esa diversidad, incluyendo las que ocurren en una misma sociedad:

Arcaísmos lingüísticos como me parece y mepiensa se pueden oír todavía hoy en el inglés actual. ${ }^{19}$

Es notable la expresión del inglés antiguo me thinks, en la cual el agente es la cosa pensada y no el sujeto pensante. El comentario de Sabatini a esa expresión es "la cosa aparece para mí".

Como sucede en inglés antiguo me thinks denota el concepto que parece o aparece para mí. ${ }^{20}$

La interpretación de me thinks es que la cosa se impone al pensamiento, la cosa se hace pensar. Imaginemos intentar hacer funcionar el cogito cartesiano con esa modalidad del verbo: "la cosa se hace pensar en mí, por lo tanto yo soy"(?) Tal razonamiento parece muy extraño e inadecuado. Tal vez sea un ejemplo de cómo el aparato psíquico condiciona nuestro lenguaje y ella, a su vez, nuestra manera de pensar.

\section{Conclusiones}

Intentando hacer una contribución al estudio de la dinámica y desarrollo del yo, este escrito se concentró en el carácter de agente del mismo. Usando recursos de la

19 Linguistic archaisms such as me seems and me thinks can still be heard in present-day English. Ibid. p. 151.Recordemos que en español se dice "me parece".

20 "As it occurs in Old English me thinks actually denotes the concept it seems or appears to me." (Sabatini, 1977, p. 524) 
lingüística y de la antropología lingüística, hemos visto cómo esa agencia del yo se manifiesta en el lenguaje a través del uso de voces verbales: activa, pasiva y media. Tomando como ejemplo principal el griego, también fueron citados desarrollos paralelos en latín y en inglés.

Estos estudios lingüísticos indicaron que los cambios en las voces pueden interpretarse como cambios en la agencia del yo. Tienen importancia particular los verbos denominados "deponentes", pues en situaciones aparentemente similares, una lengua usa la voz pasiva o media donde otra usa la voz activa. La explicación de esta diferencia no es puramente lingüística, sino que implica el papel del yo y el funcionamiento del aparato psíquico.

Sin embargo, ese desarrollo del yo no sucede en un soliloquio. Por el contrario, el aparato psíquico debe presentar formas que sean coherentes con la cultura y la sociedad en las que se desarrolla el individuo.

Lo que parece más interesante es una evidencia parcial del incremento del carácter de agente del yo. En otras palabras, parecería que el yo fue, en los límites del estudio realizado, cada vez más actuante en el transcurso de la historia.

En este sentido, una pregunta que podemos hacer es: ¿sería posible una sociedad democrática sin ese desarrollo del yo? Analizando la historia griega, vemos que el bronce implicaba la extracción de los raros y caros cobre y estaño, materia de armas costosas que no podían tener las clases inferiores de una sociedad. El surgimiento de la metalurgia del hierro -más común y más barato- creó una época de crisis, la denominada "Edad Oscura" en la historia helénica. El antiguo esquema de poder se tornó inestable porque amplios sectores inferiores de la población tenían ahora acceso a las armas. Después, algunas de esas sociedades buscaron estabilidad en regímenes democráticos (en los limites de una sociedad esclavista), cambiando el papel que tenían los individuos menos pudientes en la praxis social.

Esperamos que investigaciones futuras sean realizadas sobre estas cuestiones.

\section{Bibliografía}

-Cukier, R. (2005), Palabras de Jacob Levy Moreno, Editora Agora, Madrid.

- de Carvalho, G. A. \& Pria, A. D. (2010), Incursóes pelo o conceito de sujeito nos estudos gramaticais, Soletras, Ano X, No 20, jul./ dez.2010 - Suplemento. São Gonçalo: UERJ, 2010

- de Mijolla, A., Ed. (2005), International Dictionary of Psychoanalysis, New York: Thomson Gale.

-Dodds, E. R. (1951), The Greeks and the irrational, Berkeley: University of California Press.

-Duranti, A. (2004a), Agency in language. In [Duranti, 2004b], chapter 20, (pp. 451-473).

-Duranti, A., Ed. (2004b), A companion to linguistic anthropology, Oxford:Blackwell.

- González, C. G. (2019), Os gregos e o irracional 70 anos depois: um enfoque interdisciplinar da cultura da vergonha na Ilíada e as suas consequências psicológicas.

- Kristeva, J. (1981), Le langage, cet inconnu: une initiation à la linguistique, Paris: Seuil.

-Lacan, J. (1966), Le stade du miroir comme fondateur de la fonction du Je, telle qu'elle nous est révélée, dans l'expérience psychanalytique, volume 1 of Écrits I, (pp. 93-100), Seuil: Paris.

-Lombardini, J. R. (2010), Sentido, significado y conciencia. Master's thesis, Universidad Iberoamericana Ciudad de México, Departa- 
mento de Comunicación.

-McAfee, N. (2004), Julia Kristeva. Routledge Critical Thinkers. New York: Psychology Press.

- Pontes, E. (1986), Sujeito: da sintaxe ao discurso, Editora Atica, São Paulo.

- Roth, P. (2001), The Superego. Ideas in Psychoanalysis. Duxford, Cox \& Wyman, Cambridge.

-Sabatini, R. N. (1977). The origin of "se" as impersonal and passive particles, Hispania, 60(3), 524.Madrid.

- Sabatini, R. N. (1979). The disappearance of the impersonal construction in inglish, The South Central Bulletin, 39(4), 151-153.

-Smyth, H. W. (1956), Greek Grammar. Cambridge: Harvard University Press.

- Xenofonte (1899), Premier Livre da Cyropédie, Hachette, Paris.

-Xenofonte (1960), Cyropaideia I. The Loeb Classical Library, William Heinemann, London. 